\title{
Adverse Drug Reactions Reported in the German Democratic Republic: A Retrospective Analysis of Reports to the WHO-ADR Database
}

\author{
Lise Aagaard $^{1,2,3}$, Marion Schaefer ${ }^{4}$, Ulrich Meyer ${ }^{5}$, Ebba Holme Hansen ${ }^{1,2,3}$ \\ ${ }^{1}$ Department of Pharmacology and Pharmacotherapy, Section for Social Pharmacy, \\ Faculty of Pharmaceutical Sciences, University of Copenhagen, Copenhagen, Denmark \\ ${ }^{2}$ FKL-Research Centre for Quality in Medicine Use, Copenhagen, Denmark \\ ${ }^{3}$ Danish Pharmacovigilance Research project (DANPREP), Copenhagen, Denmark \\ ${ }^{4}$ Institut für Klinische Pharmakologie, Charitè University Medicine, Berlin, Germany \\ ${ }^{5}$ Institut für Pharmazie, Ernst Moritz Arndt University, Greifswald, Germany \\ E-mail: laa@farma.ku.dk
}

Received June 11, 2011; revised July 22, 2011; accepted August 2, 2011

\begin{abstract}
The German Democratic Republic (GDR) joined the WHO collaboration on drug safety in 1983 in order to strengthen their national pharmacovigilance system. We aim to characterize adverse drug reaction (ADR) reports which were forwarded to the WHO-ADR database by the GDR health authorities, as these data is unknown to the public. ADR reports were analysed with respect to time, type of reporter, age and sex of the patient, category of ADR (System Organ Class [SOC]), seriousness and suspected medicines. The unit of analysis was one ADR. A total of 180 individual ADR reports covering 329 ADRs were forwarded from 1985 to 1990. The largest share of ADRs was reported for psychotropic medicines (23\% of total ADRs) followed by anti-infectives for systemic use (19\% of ADRs), and medicines for the cardiovascular system (16\% of ADRs). The largest share of reported ADRs was from the SOC "hepatobiliary disorders" (16\% of total ADRs), followed by the SOCs "skin and subcutaneous disorders" (14\% of total ADRs) and "blood and lymphatic disorders" (11\% of total ADRs). Approximately 10\% of ADRs were serious and included fatal cases. In conclusion, only a limited number of ADR cases occurring in the former GDR, the majority being nonserious, were located in the WHO database. However from government files we know that a large number of serious and fatal ADRs were reported, but information about these were never communicated to the public.
\end{abstract}

Keywords: Pharmacovigilance, Spontaneous Reporting Systems, VigiBase, German Democratic Republic

\section{Introduction}

During the 1960 s, in the wake of the thalidomide catastrophe in the late 1950s [1], pharmacovigilance systems were established in many western European countries, Canada and Australia [2]. The World Health Organization (WHO) established an international reporting system in 1968 and over the years an ever-increasing number of countries have joined this collaboration [3]. In the second half of the $20^{\text {th }}$ century, a separate pharmaceutical policy system was established in the previous Council for $\mathrm{Mu}$ tual Economic Assistance (Comecon) countries, but little is known to the public about the principles of this system. The German Democratic Republic (GDR) was well- known for its large production of pharmaceuticals, often copies of medications developed by pharmaceutical companies in capitalistic countries, produced in large state-owned companies which supplied the other Comecon members [4]. New medicines were introduced years later in the GDR than in western European countries, and due to safety reasons thalidomide was never marketed in the GDR. Medicines safety was of great importance to the East German politicians and to increase medicines safety in the GDR, a national ADR reporting system was established in 1964 [4-5]. Limited information about data reported to this system was communicated to the prescribers and patients, as such type of information was considered as being a state secret and only available to 
people working with drug regulation. Available product information and/or patient leaflets were of very limited quality or non-existing and difficult to get access to, as these documents were only produced in a limited number of copies [4].

Internal documents from the GDR health authorities revealed that from 1981 to 1983 spontaneous reports covering information about more than 120 deaths from use of anti-rheumatic medications containing indometacin (Metindol/Amuno), diclofenac (Rewodina), phenylbutazone (Butazolidin), aminophenazone + phenylbutazone (Wofapyrin) and the antidiarrheal Mexaform plus (phanquinon + dichlorhydroxychinolin) and dichlorhydroxychinolin (Endiaron) existed, but no restrictions for the use of these products were made [4]. For the X-ray contrast agent iomeglamic acid (Falignost) several serious cases of allergies and anaphylactic shocks were reported which led to changes in the production, as radiographic examination were frequently used as a diagnostic method in the GDR [4].

Of political reasons the GDR were not able to join the international WHO collaboration on medicines safety until 1983, despite that other Comecon countries such as Bulgaria, the Czech Republic, Poland and Romania had already joined this collaboration in the 1970s. From 1985 until the German reunification in 1990, ADR reports were forwarded to the WHO ADR database, VigiBase by the GDR health authorities [4,6-7].

We aim to characterize these ADRs reports, as these data has never been published before, and therefore would be of interest to the public.

\section{Methods}

\subsection{Design}

We retrospectively analysed all ADR reports occurring in the GDR which were reported to VigiBase from July 1985 to May 1990. ADR data were placed at the disposal of this study in anonymous form with encrypted identification of the medicine user. ADR reports were provided by the Uppsala Monitoring Centre (UMC) as CIOMS reports, and data from these reports were manually entered into Microsoft Excel. The unit of analysis was one ADR.

We analysed the reports with regard to type of reporter, age and gender of the patient, type (system organ class [SOC]) and seriousness of reported ADRs with respect to medications involved.

\subsection{Seriousness and Causality Criteria}

The severity of ADRs was rated as certain, probable or possible according to the CIOMS scale [1]. Causality was rated as established, probable, possible, improbable and not to be ascertained. Reports were later classified as being serious or non-serious according to international criteria.

\subsection{Classification of reported ADRs}

Any ADRs reported were classified according to the international classification system Medicinal Dictionary for Regulatory Activities (MedDRA) by preferred term (PT) and (SOC) [8].

\subsection{Setting}

A national ADR reporting system was established in 1964 by law by the GDR's Ministry of Health [5]. The system was managed by the "Institut für Arzneimittelwesen der DDR" (IfAr), and physicians and pharmacists were required to report ADRs [4-5].

An official reporting form ("Meldung von Schädlichen Arzneimittelwirkungen") had to be completed, and the following information was required: age and gender of the patient; severity and characteristics of the $\operatorname{ADR}(\mathrm{s})$, suspected and concomitant medicines, indication for use, dosage, treatment period, date of onset of ADR, causality assessment and other relevant information such as laboratory data if available (internal documents).

\subsection{Medicine Use in the GDR}

Medicines licensed for use in the GDR were listed in the "Arzneimittel Verzeichnis", published periodically by the IfAr and containing information about the active ingredients of available medicines, dosage, warnings, ADRs and contra-indications [6]. From 1945 to 1990 approximately 1770 different types of medicines (prescription medicine, over-the-counter medicine, complementary medicine, herbals, homeopathic medicine, allergen extracts, blood products and radioactive medications) were licensed for use in the GDR [7]. The number and assortment varied over time. In the same period, up to 57,000 different pharmaceutical products were licensed for use in West Germany [7]. In the beginning of the 1960 s, the number of medicines imported into the GDR constituted $5 \%$ of all licensed medicines in the GDR, but this share increased up to approximately $30 \%$ of all licensed medicine in 1989/1990 [6]. The imported medicines were produced by pharmaceutical companies located in COMECON member states (estimated 20\%) or in Western European countries (estimated 10\%), and imported in the GDR by the special office/pharmacy: "Beratungsbüro für Arzneimittel", which worked for the 
GDR Ministry of Health. Medicines imported from Western countries had to be paid with Western currencies, therefore the import was limited, and the aim was in most cases to substitute in the future with GDR or COMECON production [6]. Prescription medicines were free in the GDR, and over-the-counter medicines were inexpensive. Medicine prices were fixed by the state, and rarely changed from 1945 to 1990 [6].

\section{Results}

In total 180 individual ADR reports covering 329 ADRs were reported from 1985 to 1990 . The ADR reports were of high quality and very detailed with information about causality assessment and reaction outcome. Approximately $10 \%$ of reported ADRs were serious and 13 fatal cases were located (Table 1), however these ADRs were all known ADRs. Two-thirds of the total ADRs were reported by hospital physicians, $23 \%$ of ADRs by general practitioners and $12 \%$ of ADRs were reported by specialist physicians. Serious ADRs were only reported by hospital physicians. No ADRs were reported by pharmacists.

\subsection{ADRs by Age and Sex}

The majority of reported ADRs ( $88 \%$ of total) occurred in adults, followed by $6 \%$ of ADRs reported in children from ages 3 to 10 . Less than six percent of ADRs was reported in children up to two years of age and in adolescents (ages 11 - 17). Forty percent of ADRs was reported for males and sixty percent of ADRs for females.

\subsection{ADRs by Type and Seriousness}

The largest share of reported ADRs was from the SOC "hepatobiliary disorders" (16\% of total ADRs), followed by the SOCs "skin and subcutaneous disorders" (14\% of total ADRs) and "blood and lymphatic disorders" (11\% of total ADRs).

\subsection{ADRs by Medication}

Less than $10 \%$ of ADRs were reported for medications imported from non-socialistic countries. The largest share of ADRs was reported for psychotropic medicines (23\% of total ADRs) followed by anti-infectives for systemic use (19\% of ADRs), and medicines for the cardiovascular system ( $16 \%$ of ADRs).

\section{Discussion}

This is the first study to retrospectively analyse spontaneous ADR reports from the former German Democratic Republic submitted to the WHO-ADR database. From 1985 to 1990 only a selected number of ADR reports

Table 1. Characteristics of serious adverse drug reactions leading to death reported to Vigibase, German Democratic Republic, 1985-1990.

\begin{tabular}{|c|c|c|c|c|c|c|c|c|}
\hline Case no. & Year & ATC group & Medicine & Active substance & Adverse drug reaction (ADR) & Indication & Gender & Age (year) \\
\hline 1 & 1985 & M01AB05 & Rewodina & Diclofenac & Pancytopenia & Spondolysis & Female & 64 \\
\hline 2 & 1988 & P01BD01 & Tindurin & Pyrimethamine & Agranulocytosis & Perinatal condition & Male & 1 \\
\hline 3 & 1988 & N02BB02 & Analgin & Metamizole/lidocain & Agranulocytosis & Nasopharyngitis & Male & 49 \\
\hline 4 & 1988 & J01ED07 & Mebacid & Sulfamerazine & Epidermal necrolysis & Chronic bronchitis & Female & 61 \\
\hline 5 & 1988 & J01BA01 & Berlicetin & Chloramphenicol & Marrow depression & Chronic bronchitis & Female & 49 \\
\hline 7 & 1989 & J01CA01 & Ampicillin & Ampicillin & Anaphylactic shock & Chronic bronchitis & Female & 52 \\
\hline 8 & 1989 & M01AA06 & Ketazone & Kebuzone & $\begin{array}{c}\text { Purpura } \\
\text { Thrombocytopenia }\end{array}$ & Effusion of joint & Female & 74 \\
\hline 9 & 1988 & $\begin{array}{l}\text { N02BB04 } \\
\text { M01AA06 }\end{array}$ & $\begin{array}{l}\text { Eufibron } \\
\text { Ketazone }\end{array}$ & $\begin{array}{l}\text { Propyphenazone } \\
\text { Kebuzone }\end{array}$ & $\begin{array}{c}\text { Disseminated intravascular } \\
\text { coagulation } \\
\text { Marrow depression } \\
\text { Cardiomyopathy }\end{array}$ & NA & Male & 17 \\
\hline 10 & 1989 & P01AB01 & Clont & Metronidazole & Anaphylactic shock & NA & Female & 51 \\
\hline 11 & 1990 & V08AA01 & Visotrast & Amidotrizoic acid & Anaphylactic shock & Hydronephrosis & Male & 68 \\
\hline 12 & 1990 & M03CA01 & Pavulon & Pancuronium & $\begin{array}{l}\text { Bradycardia } \\
\text { Cardiac arrest }\end{array}$ & NA & Male & $5 \mathrm{mo}$. \\
\hline 13 & 1990 & N05AH02 & Alemoxan & Clozapine & Agranulocytosis & NA & Male & 50 \\
\hline
\end{tabular}

NA: not *vailable; mo: months. 
were forwarded to VigiBase despite that around 400 to 500 ADR reports were reported annually to the GDR health authorities [4]. The low number of reports submitted to VigiBase was due to the fact that when the GDR joined the WHO collaboration the IfAr decided that only unknown and severe ADRs should be reported to VigiBase. The ADR reports located in VigiBase were in line with observations from other western European countries with respect to type and suspected medication [9-10]. Few serious cases were reported, however causality was only established in three cases. Only a small number of serious ADRs was reported in the GDR, however, several cases of hepatitis and anaphylactic shock were classified as non-serious although causality was rated as being certain or possible. In the cases of anaphylactic shock the reported medicines were often administered intravenously (IV) or subcutaneously (SC). Only one case of pancytopenia reported for diclofenac was located despite that many other serious ADR cases were reported in the 1970s and 1980s [4].

As we only have ADR data from the last six years of the existence of the GDR, it was not possible to conduct a time trend analysis. The UMC could not guarantee that the actual number of ADR reports from the GDR authorities had been higher, but reports included in this study only represent those reported to VigiBase. In general, the ADR reports were of high quality and thorough, but due to their low number, they only represent a limited number of the ADRs which eventually occurred in the former GDR. To explore whether a greater number of ADRs was reported elsewhere in East Germany, one would need access to the archives from the former East Germany.

\section{Conclusions}

Only selected information about ADRs occurring in the former GDR was located in the WHO database. However from government files we know that a large number of serious and fatal ADR cases were reported to national authorities, but information was never communicated to the patients or prescribers.

\section{References}

[1] E. B. Andrews and R. D. Mann, "Pharmacovigilance," 2nd Edition, John Wiley \& Sons, Chichester, 2007.

[2] W. H. Wardell, G. P. Velo and N. M, Jarocha, "Drug Development, Regulatory Assessment and Post Marketing Surveillance," Plenum, New York, 1981.

[3] S. Olsson, "The Role of the WHO Programme on International Drug Monitoring in Coordination Worldwide Drug Safety Efforts," Drug Safety, Vol. 19, No. 1, 1998, pp. 1-10. doi:10.2165/00002018-199819010-00001

[4] Ministerium für Gesundheitswesen. Arbeitsgruppe für Organisation und Inspektion beim Ministerrat, Kontrolabteilung. Bericht über Stand und erforderliche Massnahmen zur weiteren Erhöhung der Arzneimittelsicherheit in der DDR. Pharmazie und Technik. Berlin, 1984.

[5] J. Richter and M. Wolski, "40 Jahre Regelung und Überwa Chung des Arzneitmittelverkehrs in der Deutschen Demokratischen Republik," Pharmazie, Vol. 44, No. 10, 1989, pp. 666-671.

[6] M. Böhm, K. Gerccke, L. Kny, H. Probst and J. Richter, 45 Jahre Pharmazie in Deutschland Ost. 7bDirekt Apothekenservice AG, Fürstenfeldbruck, Berlin, 2007.

[7] J. Richter, H. J. Seidelin, M. Böhm, H. G. Keune and E. Gueinzius, "Arzneimittel-und Apothekenrecht der Republic," Akademie-Verlag, Berlin, 1985.

[8] The Medical Dictionary for Regulatory Activities (MedDRA). http://www.meddramsso.com

[9] L. Aagaard, L. H. Nielsen and E. H. Hansen, "Consumer Reporting of Adverse Drug Reactions: A Retrospective Analysis of the Danish Adverse Drug Reaction Database from 2004 to 2006," Drug Safety, Vol. 32, No. 11, 2009, pp. 1067-1074. doi:10.2165/11316680-000000000-00000

[10] J. de Langen, F. van Hunsel, A. Passier, L. de Jong-van den Berg, K. van Grootheest, "Adverse Drug Reaction Reporting by Patients in the Netherlands: Three Years of Experience," Drug Safety, Vol. 31, No. 6, 2008, 515-524. doi:10.2165/00002018-200831060-00006 


\section{Supporting Information}

Additional supporting information may be found in the online version of this article in Table S1.

Table S1. Characteristics of adverse drug reaction reports from the German Democratic Republic (GDR) to Vigibase, 1985 to 1990.

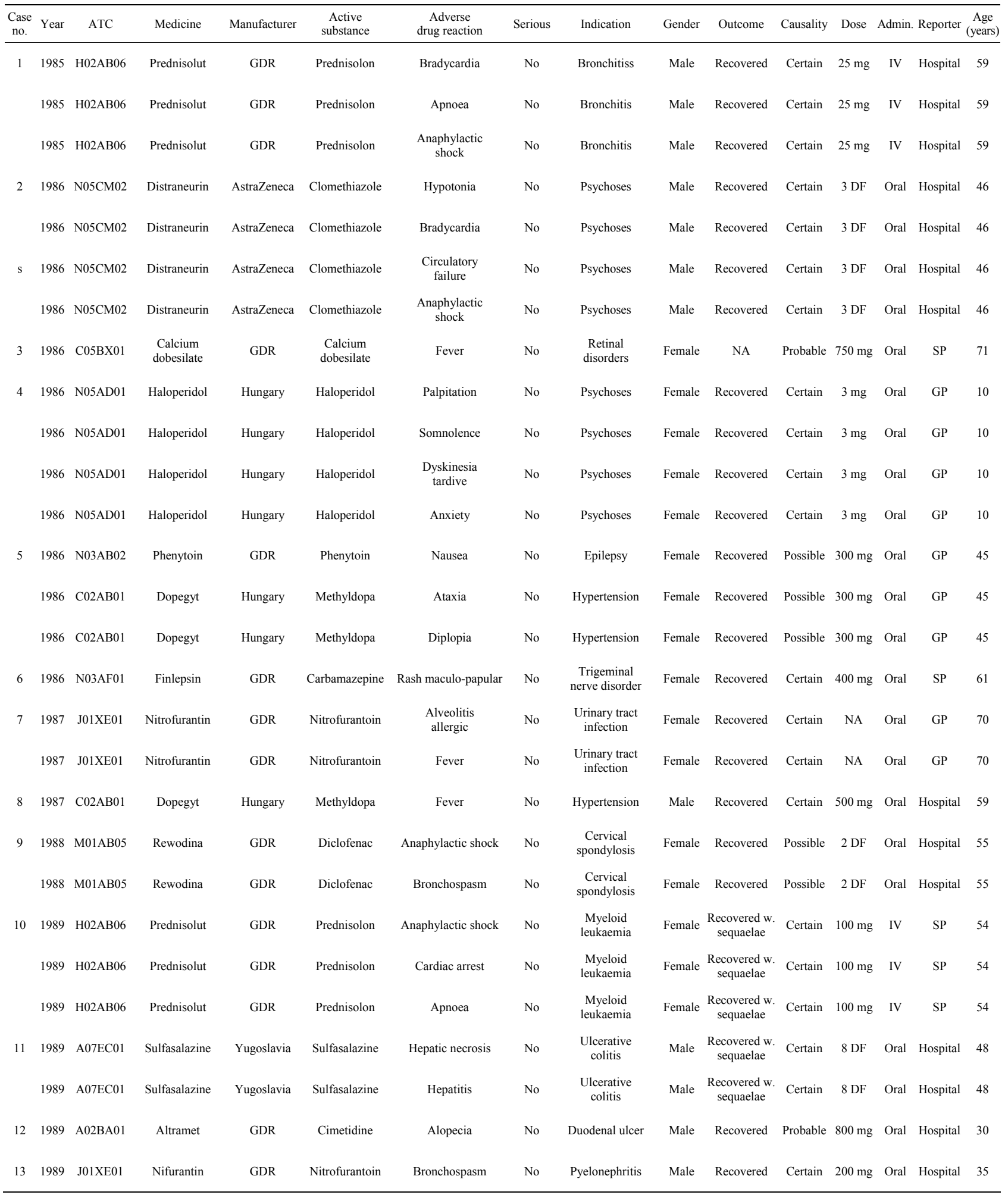




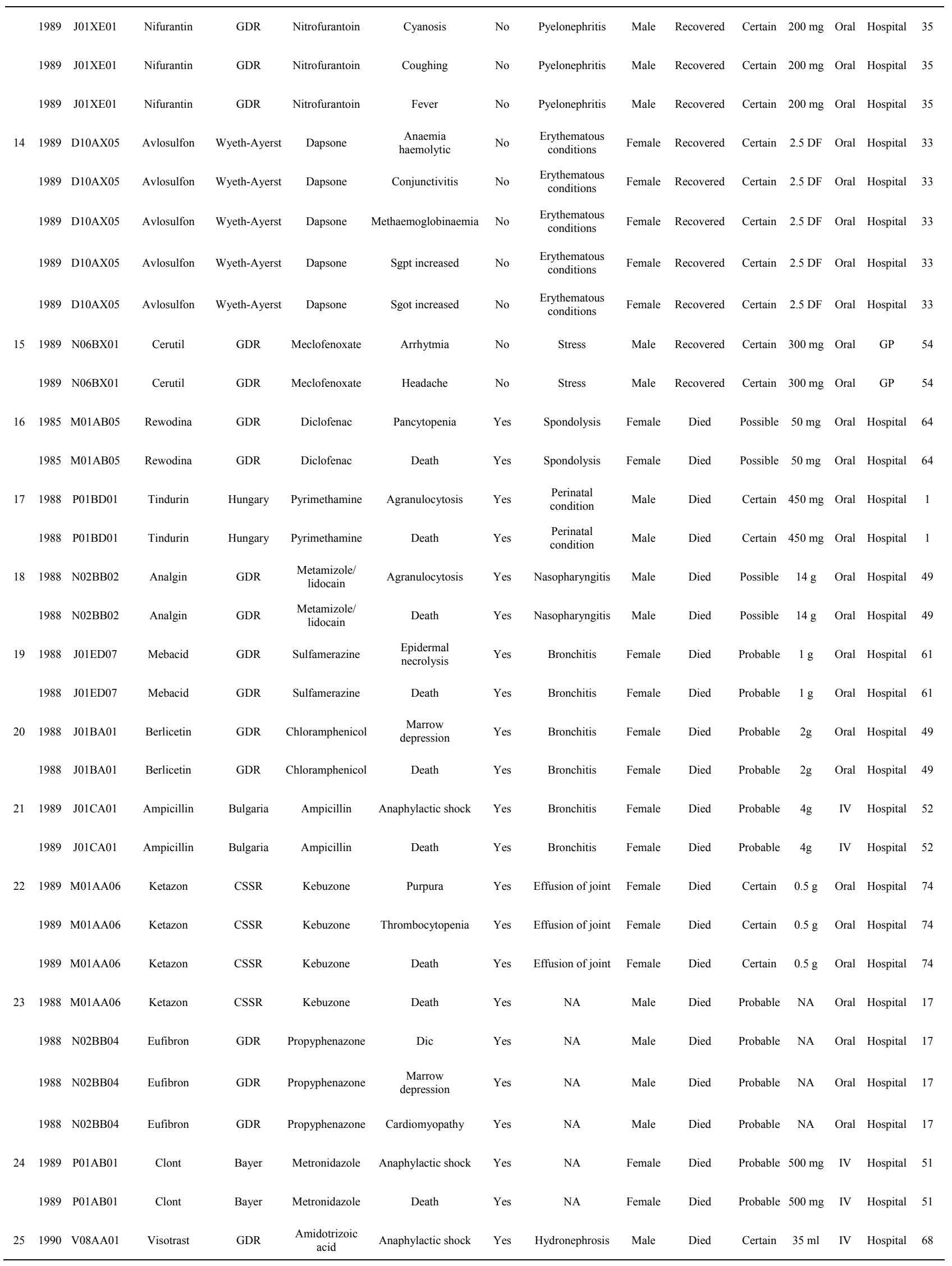




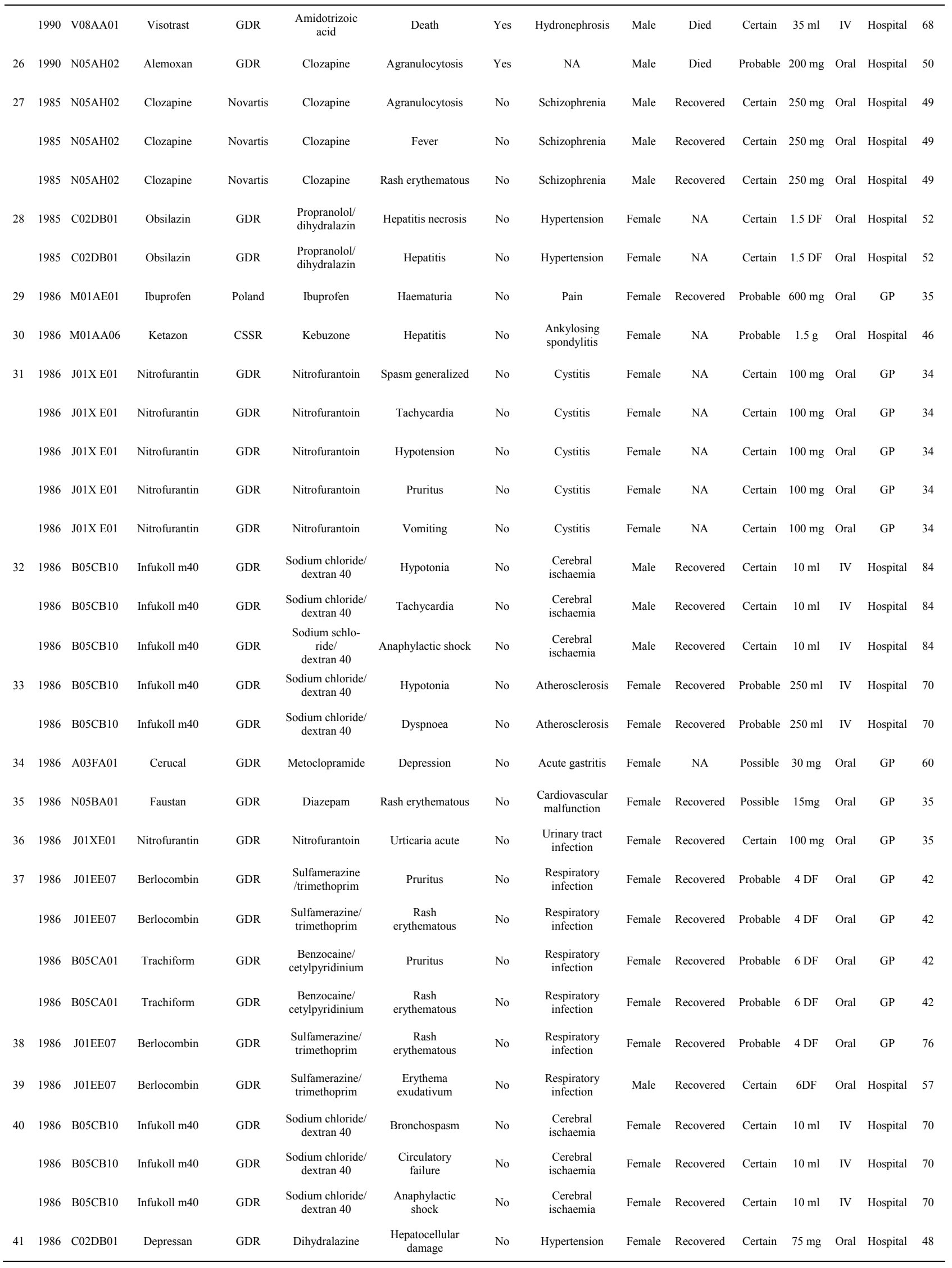




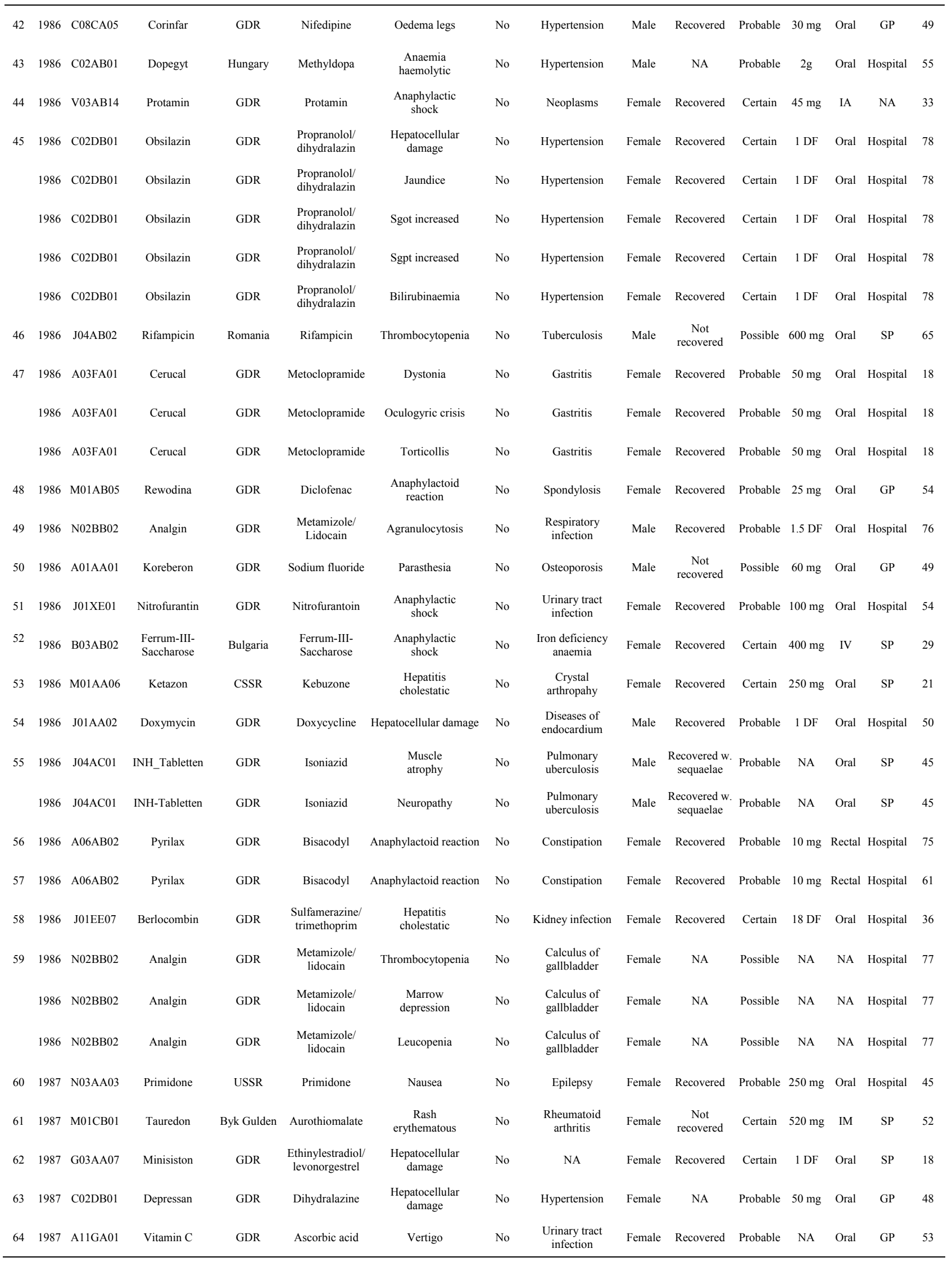




\begin{tabular}{|c|c|c|c|c|c|c|c|c|c|c|c|c|c|c|c|}
\hline & 1987 & A11GA01 & Vitamin C & GDR & Ascorbic acid & $\begin{array}{l}\text { Coordination } \\
\text { abnormal }\end{array}$ & No & $\begin{array}{l}\text { Urinary tract } \\
\text { infection }\end{array}$ & Female & Recovered & Probable & NA & Oral & GP & 53 \\
\hline 65 & 1987 & J01MB02 & Negram & Yugoslavia & Nalidixic acid & Rash erythematous & No & Kidney infection & Male & Recovered & Probable & $4 \mathrm{DF}$ & Oral & SP & 78 \\
\hline \multirow[t]{3}{*}{66} & 1986 & M01AB05 & Rewodina & GDR & Diclofenac & Vertigo & No & Osteoarthrosis & Female & $\begin{array}{l}\text { Not } \\
\text { recovered }\end{array}$ & Possible & $150 \mathrm{mg}$ & Oral & GP & 77 \\
\hline & 1986 & N04BA01 & Dopaflex & Hungary & Levodopa & Oedema legs & No & Parkinson's disease & Female & $\begin{array}{l}\text { Not } \\
\text { recovered }\end{array}$ & Possible & $4 \mathrm{DF}$ & Oral & GP & 77 \\
\hline & 1986 & N04BA01 & Dopaflex & Hungary & Levodopa & Catatonic reaction & No & Parkinson's disease & Female & $\begin{array}{c}\text { Not } \\
\text { recovered }\end{array}$ & Possible & $4 \mathrm{DF}$ & Oral & GP & 77 \\
\hline \multirow[t]{2}{*}{67} & 1986 & N04BA01 & Dopaflex & Hungary & Levodopa & Haemorrhage nos & No & Parkinson's disease & Female & Recovered & Probable & $0.5 \mathrm{mg}$ & Oral & SP & 77 \\
\hline & 1986 & N04BA01 & Dopaflex & Hungary & Levodopa & Thrombocytopenia & No & Parkinson's disease & Female & Recovered & Probable & $0.5 \mathrm{mg}$ & Oral & SP & 77 \\
\hline 68 & 1987 & G03BA02 & Oral-turinabol & GDR & Testosterone & Hepatitis & No & $\begin{array}{l}\text { Nutritional } \\
\text { deficiencies }\end{array}$ & Male & Recovered & Probable & $15 \mathrm{mg}$ & Oral & Hospital & 70 \\
\hline \multirow[t]{3}{*}{70} & 1987 & N06BX01 & Cerutil & GDR & Meclofenoxate & Tachycardia & No & $\begin{array}{l}\text { Cerebrovascular } \\
\text { disease }\end{array}$ & Female & Recovered & Possible & $500 \mathrm{mg}$ & IV & Hospital & 56 \\
\hline & 1987 & N06BX01 & Cerutil & GDR & Meclofenoxate & Parasthesia & No & $\begin{array}{c}\text { Cerebrovascular } \\
\text { disease }\end{array}$ & Female & Recovered & Possible & $500 \mathrm{mg}$ & IV & Hospital & 56 \\
\hline & 1987 & N06BX01 & Cerutil & GDR & Meclofenoxate & Nausea & No & $\begin{array}{c}\text { Cerebrovascular } \\
\text { disease }\end{array}$ & Female & Recovered & Possible & $500 \mathrm{mg}$ & IV & Hospital & 56 \\
\hline \multirow[t]{3}{*}{71} & 1987 & N06BX01 & Cerutil & GDR & Meclofenoxate & Tachycardia & No & $\begin{array}{c}\text { Cerebrovascular } \\
\text { disease }\end{array}$ & Female & Recovered & Possible & $500 \mathrm{mg}$ & IV & Hospital & 67 \\
\hline & 1987 & N06BX01 & Cerutil & GDR & Meclofenoxate & Parasthesia & No & $\begin{array}{c}\text { Cerebrovascular } \\
\text { disease }\end{array}$ & Female & Recovered & Possible & $500 \mathrm{mg}$ & IV & Hospital & 67 \\
\hline & 1987 & N06BX01 & Cerutil & GDR & Meclofenoxate & Nausea & No & $\begin{array}{c}\text { Cerebrovascular } \\
\text { disease }\end{array}$ & Female & Recovered & Possible & $500 \mathrm{mg}$ & IV & Hospital & 67 \\
\hline 72 & 1987 & P01BA01 & Chlorochin & GDR & Chloroquine & Amnesia & No & Lichen & Female & Recovered & Possible & $250 \mathrm{mg}$ & Oral & Hospital & 49 \\
\hline 73 & 1987 & H03BВ02 & Thiamazole & Merck & Thiamazole & $\begin{array}{l}\text { Hepatitis } \\
\text { cholestatic }\end{array}$ & No & Thyrotoxicosis & Female & Recovered & Probable & $30 \mathrm{mg}$ & Oral & Hospital & 36 \\
\hline \multirow[t]{5}{*}{74} & 1987 & P03AB02 & Hch-Salbe & GDR & Lindane & Convulsions local & No & Scabies & Male & Recovered & Probable & $80 \mathrm{~g}$ & Top. & Hospital & 20 \\
\hline & 1987 & Р03AB02 & Hch-Salbe & GDR & Lindane & Mydriasis & No & Scabies & Male & Recovered & Probable & $80 \mathrm{~g}$ & Top. & Hospital & 20 \\
\hline & 1987 & Р03AB02 & Hch-Salbe & GDR & Lindane & Tremor & No & Scabies & Male & Recovered & Probable & $80 \mathrm{~g}$ & Top. & Hospital & 20 \\
\hline & 1987 & Р03AB02 & Hch-Salbe & GDR & Lindane & $\begin{array}{l}\text { Sweating } \\
\text { increased }\end{array}$ & No & Scabies & Male & Recovered & Probable & $80 \mathrm{~g}$ & Top. & Hospital & 20 \\
\hline & 1987 & Р03AB02 & Hch-Salbe & GDR & Lindane & Tachycardia & No & Scabies & Male & Recovered & Probable & $80 \mathrm{~g}$ & Top. & Hospital & 20 \\
\hline \multirow[t]{3}{*}{75} & 1988 & N03AF01 & Finlepsin & GDR & Carbamazepine & $\begin{array}{l}\text { Hepatocellular } \\
\text { damage }\end{array}$ & No & Epilepsy & Male & NA & Probable & $1 \mathrm{DF}$ & Oral & GP & 28 \\
\hline & 1988 & N03AF01 & Finlepsin & GDR & Carbamazepine & Vomiting & No & Epilepsy & Male & NA & Probable & $1 \mathrm{DF}$ & Oral & GP & 28 \\
\hline & 1988 & N03AF01 & Finlepsin & GDR & Carbamazepine & Weight decrease & No & Epilepsy & Male & NA & Probable & $1 \mathrm{DF}$ & Oral & GP & 28 \\
\hline 76 & 1988 & M01AA06 & Ketazon & CSSR & Kebuzone & $\begin{array}{l}\text { Hepatitis } \\
\text { cholestatic }\end{array}$ & No & Gout & Male & Not recovered & Probable & $9 \mathrm{DF}$ & Oral & Hospital & 59 \\
\hline \multirow[t]{2}{*}{77} & 1988 & N03AF01 & Finlepsin & GDR & Carbamazepine & $\begin{array}{c}\text { Rash } \\
\text { erythematous }\end{array}$ & No & Epilepsy & Female & Recovered & Probable & $150 \mathrm{mg}$ & Oral & Hospital & 5 \\
\hline & 1988 & N03AF01 & Finlepsin & GDR & Carbamazepine & $\begin{array}{l}\text { Epidermal } \\
\text { necrolysis }\end{array}$ & No & Epilepsy & Female & Recovered & Probable & $150 \mathrm{mg}$ & Oral & Hospital & 5 \\
\hline 78 & 1988 & C05BX01 & $\begin{array}{l}\text { Calcium } \\
\text { dobesilate }\end{array}$ & GDR & $\begin{array}{l}\text { Calcium } \\
\text { dobesilate }\end{array}$ & Angioedema & No & Phlebitis & Female & Recovered & Probable & $2 \mathrm{DF}$ & Oral & SP & 42 \\
\hline \multirow[t]{2}{*}{79} & 1988 & J04AC01 & INH-Tabletten & GDR & Isoniazid & Petechiae & No & Tuberculosis & Male & NA & Probable & $3 \mathrm{DF}$ & Oral & GP & 44 \\
\hline & 1988 & J04AC01 & INH-Tabletten & GDR & Isoniazid & Oedema legs & No & Tuberculosis & Male & NA & Probable & $3 \mathrm{DF}$ & Oral & GP & 44 \\
\hline
\end{tabular}




\begin{tabular}{|c|c|c|c|c|c|c|c|c|c|c|c|c|c|c|c|}
\hline & 1988 & J04AC01 & INH-Tabletten & GDR & Isoniazid & Circulatory failure & No & Tuberculosis & Male & NA & Probable & $3 \mathrm{DF}$ & Oral & GP & 44 \\
\hline & 1988 & J04AC01 & INH-Tabletten & GDR & Isoniazid & Dyspnoea & No & Tuberculosis & Male & NA & Probable & $3 \mathrm{DF}$ & Oral & GP & 44 \\
\hline & 1988 & J04AC01 & INH-Tabletten & GDR & Isoniazid & $\begin{array}{c}\text { Rash } \\
\text { erythematous }\end{array}$ & No & Tuberculosis & Male & NA & Probable & $3 \mathrm{DF}$ & Oral & GP & 44 \\
\hline & 1988 & J04AC01 & INH-Tabletten & GDR & Isoniazid & Fever & No & Tuberculosis & Male & NA & Probable & $3 \mathrm{DF}$ & Oral & GP & 44 \\
\hline \multirow[t]{3}{*}{80} & 1988 & M01AB05 & Rewodina & GDR & Diclofenac & Bronchospasm & No & Sciatica & Male & NA & Certain & $2 \mathrm{DF}$ & Oral & Hospital & 44 \\
\hline & 1988 & M01AB05 & Rewodina & GDR & Diclofenac & Cyanosis & No & Sciatica & Male & NA & Certain & $2 \mathrm{DF}$ & Oral & Hospital & 44 \\
\hline & 1988 & M01AB05 & Rewodina & GDR & Diclofenac & Dyspnoea & No & Sciatica & Male & NA & Certain & $2 \mathrm{DF}$ & Oral & Hospital & 44 \\
\hline 81 & 1988 & N02BB02 & Analgin & GDR & $\begin{array}{l}\text { Metamizole/ } \\
\text { lidocain }\end{array}$ & $\begin{array}{l}\text { Anaphylactoid } \\
\text { reaction }\end{array}$ & No & Nasopharyngitis & Male & Recovered & Probable & $500 \mathrm{mg}$ & Oral & GP & 36 \\
\hline 83 & 1988 & M01AA06 & Ketazon & CSSR & Kebuzone & Hepatitis & No & Lumbalgia & Female & Recovered & Probable & $500 \mathrm{mg}$ & Oral & Hospital & 60 \\
\hline 84 & 1988 & B02BA01 & Kanavit & GDR & Phytomenadione & $\begin{array}{l}\text { Anaphylactic } \\
\text { shock }\end{array}$ & No & $\begin{array}{l}\text { Coagulation factor } \\
\text { deficiency }\end{array}$ & Female & Recovered & Certain & $20 \mathrm{mg}$ & IV & Hospital & 56 \\
\hline 85 & 1988 & N02BB02 & Analgin & GDR & $\begin{array}{l}\text { Metamizole/ } \\
\text { lidocain }\end{array}$ & $\begin{array}{l}\text { Anaphylactic } \\
\text { shock }\end{array}$ & No & $\begin{array}{l}\text { Respiratory } \\
\text { infection }\end{array}$ & Male & Recovered & Probable & $750 \mathrm{mg}$ & Oral & GP & 57 \\
\hline 86 & 1988 & $\mathrm{C} 02 \mathrm{AB} 01$ & Dopegyt & Hungary & Methyldopa & Hepatitis & No & Hypertension & Female & Recovered & Certain & $500 \mathrm{mg}$ & Oral & Hospital & 48 \\
\hline 87 & 1988 & C02DB01 & Depressan & GDR & Dihydralazine & Hepatitis & No & Hypertension & Female & Recovered & Probable & $25 \mathrm{mg}$ & Oral & Hospital & 27 \\
\hline 88 & 1988 & C02DB01 & Depressan & GDR & Dihydralazine & Hepatitis & No & Hypertension & Male & Recovered & Probable & $75 \mathrm{mg}$ & Oral & Hospital & 46 \\
\hline 89 & 1988 & C02DB01 & Depressan & GDR & Dihydralazine & Hepatitis & No & Hypertension & Female & Recovered & Certain & $75 \mathrm{mg}$ & Oral & Hospital & 61 \\
\hline \multirow{2}{*}{90} & 1988 & H02AB06 & Prednisolut & GDR & Prednisolon & Vomiting & No & Asthma & Female & Recovered & Certain & $50 \mathrm{mg}$ & IV & SP & 24 \\
\hline & 1988 & H02AB06 & Prednisolut & GDR & Prednisolon & $\begin{array}{c}\text { Rash } \\
\text { erythematous }\end{array}$ & No & Asthma & Female & Recovered & Certain & $50 \mathrm{mg}$ & IV & SP & 24 \\
\hline \multirow[t]{2}{*}{91} & 1988 & M01AC01 & Piroxicam & GDR & Piroxicam & $\begin{array}{c}\text { Rash } \\
\text { erythematous }\end{array}$ & No & $\begin{array}{c}\text { Rheumatoid } \\
\text { arthritis }\end{array}$ & Female & $\begin{array}{c}\text { Not } \\
\text { recovered }\end{array}$ & Probable & $40 \mathrm{mg}$ & Oral & GP & 46 \\
\hline & 1988 & M01AC01 & Piroxicam & GDR & Piroxicam & Pruritus & No & $\begin{array}{c}\text { Rheumatoid } \\
\text { arthritis }\end{array}$ & Female & $\begin{array}{c}\text { Not } \\
\text { recovered }\end{array}$ & Probable & $40 \mathrm{mg}$ & Oral & GP & 46 \\
\hline \multirow[t]{4}{*}{92} & 1988 & R05DB04 & Nullatuss & GDR & Isoaminile & Dizziness & No & Cough & Female & Recovered & Possible & $20 \mathrm{gtt}$ & Oral & GP & 30 \\
\hline & 1988 & R05DB04 & Nullatuss & GDR & Isoaminile & Headache & No & Cough & Female & Recovered & Possible & $20 \mathrm{gtt}$ & Oral & GP & 30 \\
\hline & 1988 & R05DB04 & Nullatuss & GDR & Isoaminile & $\begin{array}{l}\text { Sweating } \\
\text { increased }\end{array}$ & No & Cough & Female & Recovered & Possible & $20 \mathrm{gtt}$ & Oral & GP & 30 \\
\hline & 1988 & R05DB04 & Nullatuss & GDR & Isoaminile & Nausea & No & Cough & Female & Recovered & Possible & $20 \mathrm{gtt}$ & Oral & GP & 30 \\
\hline 93 & 1988 & J01MB02 & Negram & Yugoslavia & Nalidixic acid & $\begin{array}{c}\text { Vision } \\
\text { abnormal }\end{array}$ & No & $\begin{array}{c}\text { Kidney } \\
\text { infection }\end{array}$ & Female & $\begin{array}{c}\text { Not } \\
\text { recovered }\end{array}$ & Possible & $750 \mathrm{mg}$ & Oral & SP & 12 \\
\hline \multirow[t]{3}{*}{94} & 1988 & M01AB05 & Rewodina & GDR & Diclofenac & Thrombocytopenia & No & $\begin{array}{l}\text { Rheumatoid } \\
\text { arthritis }\end{array}$ & Female & Recovered & Possible & $75 \mathrm{mg}$ & Oral & GP & 65 \\
\hline & 1988 & M01AB05 & Rewodina & GDR & Diclofenac & Purpura & No & $\begin{array}{l}\text { Rheumatoid } \\
\text { arthritis }\end{array}$ & Female & Recovered & Possible & $75 \mathrm{mg}$ & Oral & GP & 65 \\
\hline & 1988 & M01AB05 & Rewodina & GDR & Diclofenac & Heamaturia & No & $\begin{array}{l}\text { Rheumatoid } \\
\text { arthritis }\end{array}$ & Female & Recovered & Possible & $75 \mathrm{mg}$ & Oral & GP & 65 \\
\hline \multirow[t]{2}{*}{95} & 1988 & J01FA01 & Lubomycin-b & Poland & Erythromycin & $\begin{array}{l}\text { Hepatitis } \\
\text { cholestatic }\end{array}$ & No & $\begin{array}{c}\text { Diseases of } \\
\text { sebaceous glands }\end{array}$ & Female & Recovered & Probable & $800 \mathrm{mg}$ & Oral & Hospital & 18 \\
\hline & 1988 & J01FA01 & Lubomycin-b & Poland & Erythromycin & Fever & No & $\begin{array}{c}\text { Diseases of } \\
\text { sebaceous glands }\end{array}$ & Female & Recovered & Probable & $800 \mathrm{mg}$ & Oral & Hospital & 18 \\
\hline 96 & 1988 & C02DB01 & Depressan & GDR & Dihydralazine & Thrombocytopenia & No & Hypertension & Male & Recovered & Probable & $37 \mathrm{mg}$ & Oral & Hospital & 55 \\
\hline
\end{tabular}




\begin{tabular}{|c|c|c|c|c|c|c|c|c|c|c|c|c|c|c|c|}
\hline & 1988 & C02DB01 & Depressan & GDR & Dihydralazine & Haematuria & No & Hypertension & Male & Recovered & Probable & $37 \mathrm{mg}$ & Oral & Hospital & 55 \\
\hline & 1988 & C02DB01 & Depressan & GDR & Dihydralazine & Purpura & No & Hypertension & Male & Recovered & Probable & $37 \mathrm{mg}$ & Oral & Hospital & 55 \\
\hline & 1988 & $\mathrm{C} 02 \mathrm{DB} 01$ & Depressan & GDR & Dihydralazine & Melaena & No & Hypertension & Male & Recovered & Probable & $37 \mathrm{~m}$ & Oral & Hospital & 55 \\
\hline 97 & 1988 & C07FA05 & Obsilazin & GDR & $\begin{array}{l}\text { Propranolol/ } \\
\text { dihydralazin }\end{array}$ & Hepatitis & No & Hypertension & Female & Recovered & Certain & $0.5 \mathrm{DF}$ & Oral & Hospital & 59 \\
\hline 98 & 1988 & J01FA01 & Lubomycin-b & Poland & Erythromycin & $\begin{array}{l}\text { Hepatitis } \\
\text { cholestatic }\end{array}$ & No & $\begin{array}{c}\text { Acne of } \\
\text { sebaceous glands }\end{array}$ & Female & Recovered & Probable & $800 \mathrm{mg}$ & Oral & Hospital & 23 \\
\hline 99 & 1988 & J01CE01 & Jenacillin-a & GDR & Benzylpenicillin & $\begin{array}{c}\text { Rash } \\
\text { erythematous }\end{array}$ & No & Bronchopneumonia & Male & Recovered & Probable & $800 \mathrm{kIU}$ & IM & Hospital & 2 \\
\hline 100 & 1988 & B01AA04 & Falithrom & GDR & Phenprocoumon & Hepatitis & No & $\begin{array}{l}\text { Venous } \\
\text { embolism }\end{array}$ & Female & Recovered & Probable & $3 \mathrm{mg}$ & Oral & Hospital & 37 \\
\hline 101 & 1988 & A07EC01 & Sulfasalazin & Yugoslavia & Sulfasalazine & Pruritus & No & Ulcerative colitis & Male & Recovered & Probable & $3 \mathrm{~g}$ & Oral & Hospital & 72 \\
\hline \multirow[t]{2}{*}{103} & 1988 & $\mathrm{~A} 02 \mathrm{BX} 05$ & $\begin{array}{c}\text { Pulvis } \\
\text { stomachicus SR }\end{array}$ & GDR & Bismuth & Neuropathy & No & Ulcerative colitis & Male & $\begin{array}{l}\text { Not } \\
\text { recovered }\end{array}$ & Certain & $36 \mathrm{~g}$ & Oral & Hospital & 48 \\
\hline & 1988 & $\mathrm{~A} 02 \mathrm{BX} 05$ & $\begin{array}{c}\text { Pulvis } \\
\text { stomachicus SR }\end{array}$ & GDR & Bismuth & Ataxia & No & Ulcerative colitis & Male & $\begin{array}{l}\text { Not } \\
\text { recovered }\end{array}$ & Certain & $36 \mathrm{~g}$ & Oral & Hospital & 48 \\
\hline 104 & 1988 & Н03ВВ02 & Methimazole & GDR & Thiamazole & Agranulocytosis & No & Toxic diffuse goiter & Female & Recovered & Certain & $30 \mathrm{mg}$ & Oral & Hospital & 28 \\
\hline 105 & 1988 & J01EE07 & Berlocombin & GDR & $\begin{array}{l}\text { Sulfamerazine/ } \\
\text { trimethoprim }\end{array}$ & Flushing & No & Kidney infection & Female & Recovered & Probable & $4 \mathrm{DF}$ & Oral & GP & 48 \\
\hline 106 & 1988 & N07BB01 & Disulfiram & GDR & Disulfiram & Hepatitis & No & $\begin{array}{l}\text { Alcohol } \\
\text { dependence } \\
\text { syndrome }\end{array}$ & Female & Recovered & Certain & $250 \mathrm{mg}$ & Oral & Hospital & 26 \\
\hline 107 & 1988 & C02DB01 & Obsilazin & GDR & $\begin{array}{l}\text { Propranolol/ } \\
\text { dihydralazin }\end{array}$ & Hepatitis & No & Hypertension & Female & Not recovered & Certain & $1.5 \mathrm{DF}$ & Oral & Hospital & 46 \\
\hline 108 & 1988 & C08CA05 & Corinfar & GDR & Nifedipine & Pruritus & No & Hypertension & Female & Recovered & Certain & $60 \mathrm{mg}$ & Oral & GP & 57 \\
\hline 109 & 1998 & A10BB01 & Maninil & GDR & Glibenclamide & $\begin{array}{c}\text { Anaemia } \\
\text { haemolytic }\end{array}$ & No & Diabetes mellitus & Male & Recovered & Possible & $3 \mathrm{mg}$ & Oral & Hospital & 64 \\
\hline 110 & 1988 & A03FA01 & Cerucal & GDR & Metoclopramide & $\begin{array}{l}\text { Extrapyramidal } \\
\text { disorder }\end{array}$ & No & Gastritis & Female & Recovered & Certain & $30 \mathrm{mg}$ & Oral & SP & 46 \\
\hline \multirow[t]{3}{*}{111} & 1988 & N02BB04 & Propyphenazone & GDR & Propyphenazone & Allergic reaction & No & Nasopharyngitis & Female & Recovered & Probable & $200 \mathrm{mg}$ & Rectal & Hospital & 8 \\
\hline & 1988 & N02BB04 & Propyphenazone & GDR & Propyphenazone & Bronchospasm & No & Nasopharyngitis & Female & Recovered & Probable & $200 \mathrm{mg}$ & Rectal & Hospital & 8 \\
\hline & 1988 & N02BB04 & Propyphenazone & GDR & Propyphenazone & Pruritus & No & Nasopharyngitis & Female & Recovered & Probable & $200 \mathrm{mg}$ & Rectal & Hospital & 8 \\
\hline 112 & 1988 & J01FA01 & Lubomycin-b & Poland & Erythromycin & $\begin{array}{l}\text { Hepatocellular } \\
\text { damage }\end{array}$ & No & Bronchiti & Male & Recovered & Certain & $1.6 \mathrm{~g}$ & Oral & Hospital & 8 \\
\hline 113 & 1988 & N03AG01 & Convulsofin & GDR & Valproic acid & Pancreatitis & No & Epilepsy & Female & Recovered & Probable & $80 \mathrm{gtt}$ & Oral & Hospital & 8 \\
\hline \multirow[t]{3}{*}{114} & 1989 & A07EC01 & Sulfasalazin & Yugoslavia & Sulfasalazin & Pneumonia & No & $\begin{array}{l}\text { Regional } \\
\text { enteritis }\end{array}$ & Female & $\begin{array}{l}\text { Not } \\
\text { recovered }\end{array}$ & Probable & $3 \mathrm{~g}$ & Oral & Hospital & 34 \\
\hline & 1989 & A07EC01 & Sulfasalazin & Yugoslavia & Sulfasalazin & Glossitis & No & $\begin{array}{c}\text { Regional } \\
\text { enteritis }\end{array}$ & Female & $\begin{array}{l}\text { Not } \\
\text { recovered }\end{array}$ & Probable & $3 \mathrm{~g}$ & Oral & Hospital & 34 \\
\hline & 1989 & A07EC01 & Sulfasalazin & Yugoslavia & Sulfasalazin & Nausea & No & $\begin{array}{c}\text { Regional } \\
\text { enteritis }\end{array}$ & Female & $\begin{array}{l}\text { Not } \\
\text { recovered }\end{array}$ & Probable & $3 \mathrm{~g}$ & Oral & Hospital & 34 \\
\hline 115 & 1989 & J01AA02 & Doxycycline & GDR & Doxycycline & $\begin{array}{l}\text { Anaphylactic } \\
\text { shock }\end{array}$ & No & NA & Female & Recovered & Probable & $100 \mathrm{mg}$ & IV & Hospital & 20 \\
\hline 116 & 1989 & M01CC01 & Penicillamine & CSSR & Penicillamine & $\begin{array}{c}\text { Rash } \\
\text { erythematous }\end{array}$ & No & NA & Female & Recovered & Certain & $3 \mathrm{DF}$ & Oral & Hospital & 55 \\
\hline 117 & 1989 & NA & Patentblau v & BRD & Sulphan blue & Urticaria & No & $\begin{array}{l}\text { Neoplasm of } \\
\text { prostate }\end{array}$ & Male & Recovered & Probable & $1 \mathrm{ml}$ & $\mathrm{SC}$ & Hospital & 64 \\
\hline 118 & 1989 & $\mathrm{C} 02 \mathrm{AB} 01$ & Dopegyt & Hungary & Methyldopa & Hepatitis & No & Hypertension & Female & Recovered & Probable & $250 \mathrm{mg}$ & Oral & Hospital & 53 \\
\hline 119 & 1989 & C07FA05 & Obsilazin & GDR & $\begin{array}{l}\text { Propranolol/ } \\
\text { dihydralazin }\end{array}$ & Hepatitis & No & Hypertension & Female & Recovered & Certain & $2 \mathrm{DF}$ & Oral & Hospital & 55 \\
\hline
\end{tabular}




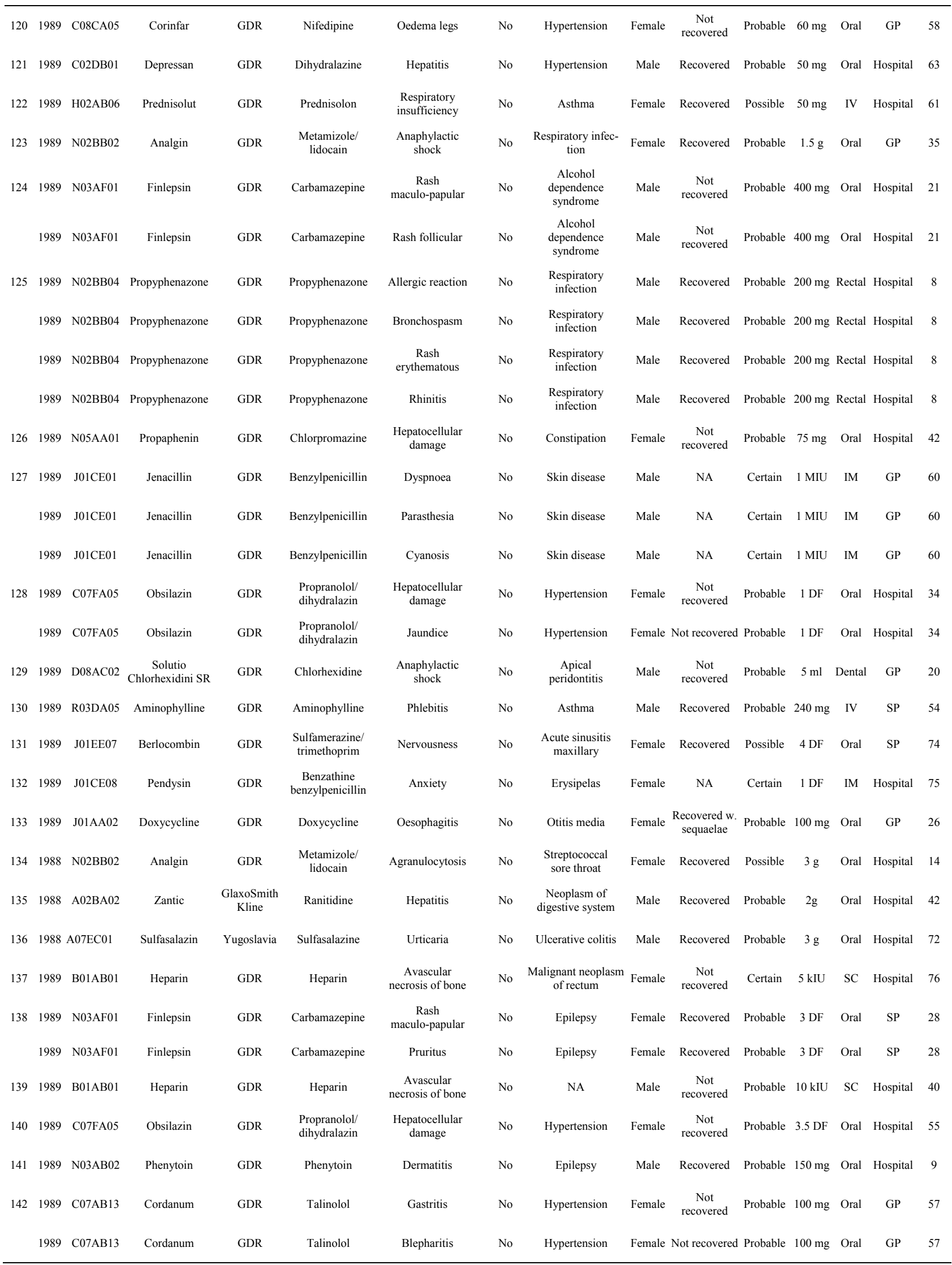




\begin{tabular}{|c|c|c|c|c|c|c|c|c|c|c|c|c|c|c|c|}
\hline & 1989 & $\mathrm{C} 07 \mathrm{AB} 13$ & Cordanum & GDR & Talinolol & Conjunctivitis & No & Hypertension & Female & Not recovered & Probable & $100 \mathrm{mg}$ & Oral & GP & 57 \\
\hline & 1989 & $\mathrm{C} 07 \mathrm{AB} 13$ & Cordanum & GDR & Talinolol & $\begin{array}{l}\text { Lacrimation } \\
\text { abnormal }\end{array}$ & No & Hypertension & Female & Not recovered & Probable & $100 \mathrm{mg}$ & Oral & GP & 57 \\
\hline & 1989 & $\mathrm{C} 07 \mathrm{AB} 13$ & Cordanum & GDR & Talinolol & $\begin{array}{c}\text { Rash } \\
\text { erythematous }\end{array}$ & No & Hypertension & Female & Not recovered & Probable & $100 \mathrm{mg}$ & Oral & GP & 57 \\
\hline & 1989 & $\mathrm{C} 07 \mathrm{AB} 13$ & Cordanum & GDR & Talinolol & Alopecia & No & Hypertension & Female & Not recovered & Probable & $100 \mathrm{mg}$ & Oral & GP & 57 \\
\hline 143 & 1989 & V08AB05 & Ultravist & Bayer & Iopromide & $\begin{array}{l}\text { Embolism } \\
\text { pulmonary }\end{array}$ & No & NA & Female & $\begin{array}{l}\text { Recovered w. } \\
\text { sequaelae }\end{array}$ & Certain & $50 \mathrm{ml}$ & IV & Hospital & 48 \\
\hline \multirow[t]{2}{*}{144} & 1989 & J01FA01 & Etromycin & Orion & Erythromycin & $\begin{array}{l}\text { Hepatocellular } \\
\text { damage }\end{array}$ & No & NA & Male & Recovered & Certain & $360 \mathrm{mg}$ & Oral & Hospital & 2 \\
\hline & 1989 & J01FA01 & Etromycin & Orion & Erythromycin & Sgpt increased & No & NA & Male & Recovered & Certain & $360 \mathrm{mg}$ & Oral & Hospital & 2 \\
\hline 145 & 1989 & N05AH02 & Leponex & Novartis & Clozapine & Agranulocytosis & No & $\begin{array}{l}\text { Paranoid type } \\
\text { schizophrenia }\end{array}$ & Female & Recovered & Probable & $200 \mathrm{mg}$ & Oral & Hospital & 24 \\
\hline 146 & 1989 & M01AA06 & Ketazon & CSSR & Kebuzone & $\begin{array}{l}\text { Hepatitis } \\
\text { cholestatic }\end{array}$ & No & Back disorder & Female & Recovered & Probable & $10 \mathrm{ml}$ & IM & Hospital & 66 \\
\hline \multirow[t]{2}{*}{147} & 1989 & M01AC01 & Piroxicam & GDR & Piroxicam & Vision abnormal & No & $\begin{array}{l}\text { Systemic lupus } \\
\text { erythematous }\end{array}$ & Male & Recovered & Probable & $20 \mathrm{mg}$ & Oral & Hospital & 43 \\
\hline & 1989 & M01AC01 & Piroxicam & GDR & Piroxicam & $\begin{array}{l}\text { Accommodation } \\
\text { abnormal }\end{array}$ & No & $\begin{array}{l}\text { Systemic lupus } \\
\text { erythematous }\end{array}$ & Male & recovered & Probable & $20 \mathrm{mg}$ & Oral & Hospital & 43 \\
\hline \multirow[t]{3}{*}{148} & 1989 & C02DB01 & Depressan & GDR & Dihydralazine & Hepatitis & No & Hypertension & Male & Recovered & Probable & $2 \mathrm{DF}$ & Oral & SP & 53 \\
\hline & 1989 & C02DB01 & Depressan & GDR & Dihydralazine & Sgot increased & No & Hypertension & Male & recovered & Probable & $2 \mathrm{DF}$ & Oral & SP & 53 \\
\hline & 1989 & C02DB01 & Depressan & GDR & Dihydralazine & Sgpt increased & No & Hypertension & Male & recovered & Probable & $2 \mathrm{DF}$ & Oral & SP & 53 \\
\hline 149 & 1989 & J01AA02 & Doxycycline & GDR & Doxycycline & Fever & No & NA & Female & Recovered & Probable & $0.1 \mathrm{~g}$ & Oral & Hospital & 40 \\
\hline 150 & 1989 & A08A A & Sedafamem & GDR & Phendimetrazine & Psychosis & No & Obesity & Male & Recovered & Certain & $2 \mathrm{DF}$ & Oral & GP & 40 \\
\hline 151 & 1989 & N03AG01 & Convulsofin & GDR & Valproic acid & Thrombocytopenia & No & Epilepsy & Male & Recovered & Certain & $3 \mathrm{DF}$ & Oral & Hospital & 12 \\
\hline 152 & 1989 & $\mathrm{C} 02 \mathrm{DB} 01$ & Depressan & GDR & Dihydralazine & $\begin{array}{l}\text { Hepatitis } \\
\text { cholestatic }\end{array}$ & No & Hypertension & Male & Recovered & Certain & $75 \mathrm{mg}$ & Oral & GP & 55 \\
\hline \multirow[t]{2}{*}{153} & 1989 & M01AA06 & Ketazon & CSSR & Kebuzone & Hepatitis & No & Niple infection & Female & Recovered & Probable & $250 \mathrm{mg}$ & Oral & Hospital & 25 \\
\hline & 1989 & M01AA06 & Ketazon & CSSR & Kebuzone & Hepatitis & No & Gout & Female & Recovered & Probable & $750 \mathrm{mg}$ & Oral & Hospital & 49 \\
\hline 154 & 1989 & B01AD01 & Awelysin & GDR & Streptokinase & Hepatitis & No & Venous embolism & Male & Recovered & Probable & $3 \mathrm{MIU}$ & IV & Oral & NA \\
\hline 155 & 1989 & B01AB01 & Heparin & GDR & Heparin & $\begin{array}{c}\text { Avascular } \\
\text { necrosis of bone }\end{array}$ & No & NA & Female & $\begin{array}{l}\text { Not } \\
\text { recovered }\end{array}$ & Probable & $10 \mathrm{kIU}$ & $\mathrm{SC}$ & Hospital & 29 \\
\hline 156 & 1989 & N02AC03 & $\begin{array}{c}\text { Tiretta } \\
\text { analgica "p" }\end{array}$ & GDR & $\begin{array}{c}\text { Codeine/ } \\
\text { papaverin/ } \\
\text { aminophenazone/ } \\
\text { phenazone }\end{array}$ & $\begin{array}{l}\text { Anaphylactic } \\
\text { shock }\end{array}$ & No & Migraine & Female & Recovered & Probable & $4 \mathrm{ml}$ & IV & GP & 26 \\
\hline 157 & 1989 & B01AD01 & Awelysin & GDR & Streptokinase & $\begin{array}{l}\text { Anaphylactic } \\
\text { shock }\end{array}$ & No & $\begin{array}{l}\text { Acute myocardial } \\
\text { infection }\end{array}$ & Male & NA & Probable & $0.2 \mathrm{MIU}$ & IV & Hospital & 47 \\
\hline 158 & 1989 & S03AA08 & Berlicetin & GDR & $\begin{array}{l}\text { Prednisolone/ } \\
\text { chloramphenicol }\end{array}$ & Otitis externa & No & Ear disorder & Female & Recovered & Certain & $1 \mathrm{ml}$ & Top. & SP & 50 \\
\hline 159 & 1989 & S03AA08 & Berlicetin & GDR & $\begin{array}{l}\text { Prednisolone/ } \\
\text { chloramphenicol }\end{array}$ & Otitis externa & No & Ear disorder & Male & Recovered & Certain & $1 \mathrm{ml}$ & Top. & SP & 33 \\
\hline \multirow{3}{*}{160} & 1989 & S03AA08 & Berlicetin & GDR & $\begin{array}{l}\text { Prednisolone/ } \\
\text { chloramphenicol }\end{array}$ & Pruritus & No & Ear disorder & Male & Recovered & Certain & $1 \mathrm{ml}$ & Top. & SP & 33 \\
\hline & 1989 & N05AH02 & Alemoxan & GDR & Clozapine & Agranulocytosis & No & Paranoid states & Female & $\begin{array}{l}\text { Not } \\
\text { recovered }\end{array}$ & Probable & $200 \mathrm{mg}$ & Oral & Hospital & 38 \\
\hline & 1989 & N05AH02 & Alemoxan & GDR & Clozapine & Rhinitis & No & Paranoid states & Female & $\begin{array}{l}\text { Not } \\
\text { recovered }\end{array}$ & Probable & $200 \mathrm{mg}$ & Oral & Hospital & 38 \\
\hline
\end{tabular}




\begin{tabular}{|c|c|c|c|c|c|c|c|c|c|c|c|c|c|c|c|}
\hline & 1989 & N05AH02 & Alemoxan & GDR & Clozapine & Bronchitis & No & Paranoid states & Female & $\begin{array}{c}\text { Not } \\
\text { recovered }\end{array}$ & Probable & $200 \mathrm{mg}$ & Oral & Hospital & 38 \\
\hline & 1989 & N05AH02 & Alemoxan & GDR & Clozapine & Rash pustular & No & Paranoid states & Female & $\begin{array}{l}\text { Not } \\
\text { recovered }\end{array}$ & Probable & $200 \mathrm{mg}$ & Oral & Hospital & 38 \\
\hline \multirow[t]{4}{*}{161} & 1989 & A07EC01 & Sulfasalazin & Yugoslavia & Sulfasalazine & $\begin{array}{c}\text { Rash } \\
\text { erythematous }\end{array}$ & No & $\begin{array}{l}\text { Ulcerative } \\
\text { colitis }\end{array}$ & Male & Recovered & Probable & $3.5 \mathrm{~g}$ & Oral & Hospital & 52 \\
\hline & 1989 & A07EC01 & Sulfasalazin & Yugoslavia & Sulfasalazine & Sgot increased & No & $\begin{array}{l}\text { Ulcerative } \\
\text { colitis }\end{array}$ & Male & Recovered & Probable & $3.5 \mathrm{~g}$ & Oral & Hospital & 52 \\
\hline & 1989 & A07EC01 & Sulfasalazin & Yugoslavia & Sulfasalazine & Sgpt increased & No & $\begin{array}{l}\text { Ulcerative } \\
\text { colitis }\end{array}$ & Male & Recovered & Probable & $3.5 \mathrm{~g}$ & Oral & Hospital & 52 \\
\hline & 1989 & A07EC01 & Sulfasalazin & Yugoslavia & Sulfasalazine & Leukocytosis & No & $\begin{array}{l}\text { Ulcerative } \\
\text { colitis }\end{array}$ & Male & Recovered & Probable & $3.5 \mathrm{~g}$ & Oral & Hospital & 52 \\
\hline \multirow[t]{2}{*}{162} & 1989 & M01CB01 & Tauredon & Byk Gulden & Aurothiomalate & Purpura & No & $\begin{array}{c}\text { Rheumatoid } \\
\text { arthritis }\end{array}$ & Female & Recovered & Certain & $220 \mathrm{mg}$ & IM & Hospital & 73 \\
\hline & 1989 & M01CB01 & Tauredon & Byk Gulden & Aurothiomalate & Trombocytopenia & No & $\begin{array}{c}\text { Rheumatoid } \\
\text { arthritis }\end{array}$ & Female & Recovered & Certain & $220 \mathrm{mg}$ & IM & Hospital & 73 \\
\hline 164 & 1989 & A02BA01 & Altramet & GDR & Cimetidine & Gynaecomastia & No & Oesophagitis & Male & Recovered & Probable & $1 \mathrm{~g}$ & Oral & Hospital & 62 \\
\hline 165 & 1989 & J01GB03 & Gentamicin & Bulgaria & Gentamicin & Deafness & No & Fracture of ribs & Male & $\begin{array}{c}\text { Not } \\
\text { recovered }\end{array}$ & Probable & $120 \mathrm{mg}$ & IV & Hospital & 48 \\
\hline 166 & 1990 & J01CE01 & Penicillin $g$ & GDR & Benzylpenicillin & $\begin{array}{l}\text { Anaphylactic } \\
\text { shock }\end{array}$ & No & NA & Female & Recovered & Certain & $5 \mathrm{ml}$ & IV & Hospital & 24 \\
\hline 167 & 1990 & N01AX04 & Sombrevin & Hungary & Propanidid & Thrombophlebitis & No & Phlebitis & Female & $\begin{array}{l}\text { Recovered w. } \\
\text { sequaelae }\end{array}$ & Probable & NA & NA & Hospital & 51 \\
\hline 168 & 1990 & N01AX04 & Sombrevin & Hungary & Propanidid & Thrombophlebitis & No & Phlebitis & Female & Recovered & Probable & NA & NA & Hospital & 38 \\
\hline 169 & 1990 & N01AX04 & Sombrevin & Hungary & Propanidid & Thrombophlebitis & No & Phlebitis & Female & Recovered & Probable & NA & NA & Hospital & 36 \\
\hline \multirow[t]{4}{*}{170} & 1990 & B01AD01 & Awelysin & GDR & Streptokinase & Dizziness & No & Phlebitis & Male & Recovered & Certain & $1 \mathrm{MIU}$ & IV & Hospital & 66 \\
\hline & 1990 & B01AD01 & Awelysin & GDR & Streptokinase & Headache & No & Phlebitis & Male & Recovered & Certain & $1 \mathrm{MIU}$ & IV & Hospital & 66 \\
\hline & 1990 & B01AD01 & Awelysin & GDR & Streptokinase & Hypertension & No & Phlebitis & Male & Recovered & Certain & $1 \mathrm{MIU}$ & IV & Hospital & 66 \\
\hline & 1990 & B01AD01 & Awelysin & GDR & Streptokinase & $\begin{array}{l}\text { Sweating } \\
\text { increased }\end{array}$ & No & Phlebitis & Male & Recovered & Certain & $1 \mathrm{MIU}$ & IV & Hospital & 66 \\
\hline 171 & 1990 & C08CA05 & Corinfar & GDR & Nifedipine & $\begin{array}{c}\text { Taste } \\
\text { perversion }\end{array}$ & No & Hypertension & Male & Recovered & Probable & NA & NA & GP & 49 \\
\hline \multirow[t]{3}{*}{172} & 1990 & J04AB02 & Rifampicin & Rumania & Rifampicin & Nausea & No & NA & Female & Recovered & Possible & NA & Oral & SP & 41 \\
\hline & 1990 & J04AB02 & Rifampicin & Rumania & Rifampicin & Purpura & No & NA & Female & Recovered & Possible & NA & Oral & SP & 41 \\
\hline & 1990 & J04AB02 & Rifampicin & Rumania & Rifampicin & Trombopenia & No & NA & Female & Recovered & Possible & NA & Oral & SP & 41 \\
\hline \multirow[t]{4}{*}{173} & 1990 & C02DB01 & Depressan & GDR & Dihydralazine & Anxiety & No & Hypertension & Female & Recovered & Probable & $25 \mathrm{mg}$ & Oral & GP & 34 \\
\hline & 1990 & C02DB01 & Depressan & GDR & Dihydralazine & Depression & No & Hypertension & Female & Recovered & Probable & $25 \mathrm{mg}$ & Oral & GP & 34 \\
\hline & 1990 & C02DB01 & Depressan & GDR & Dihydralazine & Hypertension & No & Hypertension & Female & Recovered & Probable & $25 \mathrm{mg}$ & Oral & GP & 34 \\
\hline & 1990 & C02DB01 & Depressan & GDR & Dihydralazine & Vision abnormal & No & Hypertension & Female & Recovered & Probable & $25 \mathrm{mg}$ & Oral & GP & 34 \\
\hline \multirow[t]{3}{*}{174} & 1990 & B01AB01 & Heparin & GDR & Heparin & Nausea & No & NA & Male & Recovered & Possible & $15 \mathrm{kIU}$ & $\mathrm{SC}$ & Hospital & 83 \\
\hline & 1990 & $\mathrm{~B} 01 \mathrm{AB} 01$ & Heparin & GDR & Heparin & Vision abnormal & No & NA & Male & Recovered & Possible & $15 \mathrm{kIU}$ & $\mathrm{SC}$ & Hospital & 83 \\
\hline & 1990 & B01AB01 & Heparin & GDR & Heparin & Vomiting & No & NA & Male & Recovered & Possible & $15 \mathrm{kIU}$ & $\mathrm{SC}$ & Hospital & 83 \\
\hline 175 & 1990 & J04AB02 & Rifampicin & Rumania & Rifampicin & Renal failure acute & No & NA & Male & Recovered & Possible & NA & Oral & SP & 57 \\
\hline
\end{tabular}




\begin{tabular}{|c|c|c|c|c|c|c|c|c|c|c|c|c|c|c|c|}
\hline 176 & 1990 & J01FA01 & Lubomycin-b & Poland & Erythromycin & $\begin{array}{l}\text { Hepatitis } \\
\text { cholestatic }\end{array}$ & No & NA & Female & NA & Probable & NA & Oral & Hospital & 3 \\
\hline & 1990 & J01FA01 & Lubomycin-b & Poland & Erythromycin & Hepatomegaly & No & NA & Female & NA & Probable & NA & Oral & Hospital & 3 \\
\hline & 1990 & J01FA01 & Lubomycin-b & Poland & Erythromycin & $\begin{array}{c}\text { Sgot } \\
\text { increased }\end{array}$ & No & NA & Female & NA & Probable & NA & Oral & Hospital & 3 \\
\hline & 1990 & J01FA01 & Lubomycin-b & Poland & Erythromycin & $\begin{array}{c}\text { Sgpt } \\
\text { increased }\end{array}$ & No & NA & Female & NA & Probable & NA & Oral & Hospital & 3 \\
\hline 177 & 1990 & J01CE01 & Jenacillin-a & GDR & Benzylpenicillin & Dizziness & No & Erysipelas & Male & Recovered & Probable & $2 \mathrm{MIU}$ & $\mathrm{IM}$ & Hospital & 54 \\
\hline \multirow[t]{3}{*}{178} & 1990 & N01AB01 & Halan & GDR & Halothane & $\begin{array}{l}\text { Hepatic function } \\
\text { abnormal }\end{array}$ & No & NA & Male & Recovered & Certain & 1 times & INH & Hospital & 49 \\
\hline & 1990 & N01AB01 & Halan & GDR & Halothane & Hepatitis & No & NA & Male & Recovered & Certain & 1 times & INH & Hospital & 49 \\
\hline & 1990 & N01AB01 & Halan & GDR & Halothane & Eosinophilia & No & NA & Male & Recovered & Certain & 1 times & INH & Hospital & 49 \\
\hline \multirow[t]{2}{*}{179} & 1990 & A12AA06 & $\begin{array}{l}\text { Calcium } \\
\text { dobesilate }\end{array}$ & GDR & $\begin{array}{l}\text { Calcium } \\
\text { dobesilate }\end{array}$ & $\begin{array}{l}\text { Injection site } \\
\text { reaction }\end{array}$ & No & $\begin{array}{l}\text { Abnormal blood } \\
\text { chemistry }\end{array}$ & Female & $\begin{array}{c}\text { Not } \\
\text { recovered }\end{array}$ & Certain & $20 \mathrm{ml}$ & IV & Hospital & $3 \mathrm{mo}$. \\
\hline & 1990 & A12AA06 & $\begin{array}{l}\text { Calcium } \\
\text { dobesilate }\end{array}$ & GDR & $\begin{array}{l}\text { Calcium } \\
\text { dobesilate }\end{array}$ & Calcinosis & No & $\begin{array}{l}\text { Abnormal blood } \\
\text { chemistry }\end{array}$ & Female & $\begin{array}{l}\text { Not } \\
\text { recovered }\end{array}$ & Certain & $20 \mathrm{ml}$ & IV & Hospital & $3 \mathrm{mo}$. \\
\hline \multirow[t]{2}{*}{180} & 1990 & M03AC01 & Pavulon & $\begin{array}{l}\text { Organon } \\
\text { Teknica }\end{array}$ & Pancuronium & Bradycardia & Yes & NA & Male & Died & Possible & $\mathrm{mg}$ & IC & Hospital & $5 \mathrm{mo}$. \\
\hline & 1990 & M03AC01 & Pavulon & $\begin{array}{l}\text { Organon } \\
\text { Teknica }\end{array}$ & Pancuronium & Cardiac arrest & Yes & NA & Male & Died & Possible & $.4 \mathrm{mg}$ & IC & Hospital & $5 \mathrm{mo}$. \\
\hline
\end{tabular}

\section{Abbreviations}

GP: general practitioner; SP: specialist physician; DF: defined formulation; mo.: months; IV: intravenous; IA: intraarteriel; IM: intramuscular; SC: subcutaneous; INH: inhalation; Top: topical; IC: intracardiac; BRD: Bundesrepublic Deutschland; CSSR: Czechoslovak Socialist Republic. 